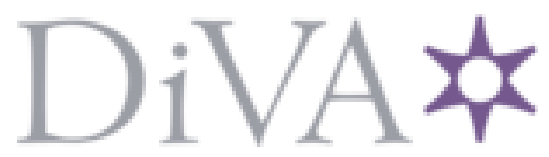

http://www.diva-portal.org

\title{
Postprint
}

This is the accepted version of a paper presented at 5th IEEE PES Asia-Pacific Power and Energy Engineering Conference.

Citation for the original published paper:

Hamon, C., Perninge, M., Söder, L. (2013)

The value of using chance-constrained optimal power flows for generation re-dispatch under uncertainty with detailed security constraints.

In: Proceedings of the 5th IEEE PES Asia-Pacific Power and Energy Engineering Conference

N.B. When citing this work, cite the original published paper.

Permanent link to this version:

http://urn.kb.se/resolve?urn=urn:nbn:se:kth:diva-14052 1 


\section{The value of using chance-constrained optimal power flows for generation re-dispatch under uncertainty with detailed security constraints}

\author{
Camille Hamon \\ Electric Power Systems \\ Royal Institute of Technology \\ Email: camille.hamon@ee.kth.se
}

\author{
Magnus Perninge \\ Department of Automatic Control \\ Lund University
}

\author{
Lennart Söder \\ Electric Power Systems \\ Royal Institute of Technology
}

\begin{abstract}
The uncertainty faced in the operation of power systems increases as larger amounts of intermittent sources, such as wind and solar power, are being installed. Traditionally, an optimal generation re-dispatch is obtained by solving securityconstrained optimal power flows (SCOPF). The resulting system operation is then optimal for given values of the uncertain parameters. New methods have been developed to consider the uncertainty directly in the generation re-dispatch optimization problem. Chance-constrained optimal power flows (CCOPF) are such methods.

In this paper, SCOPF and CCOPF are compared and the benefits of using CCOPF for power systems operation under uncertainty are discussed. The discussion is illustrated by a case study in the IEEE 39 bus system, in which the generation redispatch obtained by CCOPF is shown to always be cheaper than that obtained by SCOPF.
\end{abstract}

\section{INTRODUCTION}

The reshaping of the generation portfolio with more intermittent energy sources such as wind power brings about new challenges for power systems operation [1]. Among the new challenges brought about by larger amounts of wind power, a need for new tools which account for the entire underlying probability distribution of wind power and load has been advocated [2], [3]. In this paper, such tools are discussed in the context of generation re-dispatch during the operational time frame.

New re-dispatch algorithms which account for the probability distribution of the uncertainty, such as future levels of wind power production and load, have recently been devised [4]-[6]. They belong to the class of chance-constrained optimization problems, which are optimization problems with probabilistic constraints. In the context of power systems, they are sometimes called stochastic optimal power flows (SOPF). In the following, however, the term chance-constrained optimal power flows (CCOPF) will be used.

In [4], a chance-constrained optimal power flow formulation is given that maximizes the power transfers over a set of

The financial support for this project from Vindforsk is greatly acknowledged. M. Perninge is a member of the LCCC Linnaeus Center and the eLLIIT Excellence Center at Lund University. buses, while maintaining the probability of violation of transfer limits across specified bottlenecks under a certain value. In the same work, a CCOPF formulation for computing available transfer capacity (ATC) is given. In [5], a CCOPF formulation is given that minimizes production costs, while ensuring that the probability that the flows on certain lines exceed the transfer limits is less than a defined level. In [6], the CCOPF formulation minimizes production costs while ensuring that the probability of system failure does not exceed a specified level. Voltage stability, small-signal stability and operational limits are considered.

A challenge in the CCOPF formulations above is to compute the probabilities appearing in the probabilistic constraints, which is needed to solve the CCOPF problems. The reason is that the random variables in these constraints are functions of the uncertain parameters whose probability distributions are assumed to be known. The probability distributions of these random variables are therefore not known except in very simple cases (for example if one constraint is a linear function of only one of the uncertain parameters). In [4], it is solved by using Cornish-Fisher expansions of the linearized power transfers. In [5], the power transfers are linearized to become linear functions of the uncertain parameters, and the probabilistic constraints are back-mapped to the uncertain parameter space using these linear functions. In [6], CornishFisher expansions are also used to approximate the probability of system failure.

In the publications presented above, only Gaussian distributed random variables are included in the uncertainty. Previous research has shown, however, that wind power forecast errors are not Gaussian distributed. Instead, beta and hyperbolic distributions seem appropriate [7], [8]. In [9], the same CCOPF formulation as in [6] is used, but the solution method is extended in order to account for non-Gaussian distributions. The new method uses Edgeworth expansions instead of Cornish-Fisher expansions.

The CCOPF-based re-dispatch algorithm should be able to optimize the use of the system. While all the methods discussed above have been proven to work on case studies, 
no assessment of the value of switching from the traditional deterministic re-dispatch algorithms to these new CCOPF formulations has been made. In this paper, we aim at filling this gap by comparing the traditional re-dispatch with the CCOPF formulation in [9].

The remainder of this paper is organized as follows. Section II presents one deterministic re-dispatch algorithm, namely security-constrained optimal power flow (SCOPF). Section III reviews the CCOPF formulation from [9] and the method developed to solve it. Section IV compares the two re-dispatch methods in the IEEE 39 bus system.

\section{SECURITY-CONSTRAINED OPTIMAL POWER FLOWS}

Generation re-dispatch are performed so that the socioeconomic costs are minimized. It implies looking for the cheapest way of re-dispatching the generation in the participating generators which ensures a secure operation of the system. The system security is usually measured by the $N-1$ criterion, which states that the system must be stable after the occurrence of any pre-selected contingency. The re-dispatch problem is then formulated as a security-constrained optimal power flow (SCOPF). The SCOPF:s are either preventive [10] or corrective [11], [12]. In this work, the preventive SCOPF will be considered. It can be expressed as

$$
\begin{array}{ll}
\min _{u_{0}} & C\left(x_{0}, \hat{\zeta}, u_{0}\right) \\
\text { s.t. } & f_{i}\left(x_{i}, \hat{\zeta}, u_{0}\right)=0, \quad i=0, \ldots, n_{c} \\
& h_{i}\left(x_{i}, \hat{\zeta}, u_{0}\right) \leq 0, \quad i=0, \ldots, n_{c}
\end{array}
$$

where $x \in \mathbb{R}^{n_{x}}, \hat{\zeta} \in \mathbb{R}^{l}$ and $u_{0} \in \mathbb{R}^{n_{u}}$ are the state variables (such as voltage magnitudes and angles), the parameters such as the active power consumption and wind power production - and the control variables, respectively. The function $C: \mathbb{R}^{n_{x}} \times \mathbb{R}^{l} \times \mathbb{R}^{n_{u}} \rightarrow \mathbb{R}$ is the objective function to be minimized, $f: \mathbb{R}^{n_{x}} \times \mathbb{R}^{l} \times \mathbb{R}^{n_{u}} \rightarrow \mathbb{R}^{n_{x}}$ represents the power flow equations ensuring that the solution corresponds to a possible equilibrium, and $h: \mathbb{R}^{n_{x}} \times \mathbb{R}^{l} \times \mathbb{R}^{n_{u}} \rightarrow \mathbb{R}^{n_{l}}$ contains $n_{l}$ operational constraints. The indexes $i=1, \ldots, n_{c}$ correspond to the post-contingency systems for the $n_{c}$ pre-selected contingencies, and $i=0$ to the pre-contingency system. Contingencies can for example be the loss of generation units or transmission lines. The notation $\hat{\zeta}$ denotes that the SCOPF is solved for a certain value of the uncertain parameters $\zeta$.

A challenge with SCOPF is to choose these values $\hat{\zeta}$ that represent the situations for which the system is studied. Consider for example a system in which two of the loads $P_{1}$ and $P_{2}$ are forecasted to be Gaussian distributed with their joint probability distribution function given in Figure 1. The corresponding SCOPF problem is solved for a corresponding operating situation described by chosen values $\hat{P}_{1}$ and $\hat{P}_{2}$ of these loads. The probability distributions given by forecasts are used to choose these values.

Two issues arise:

- Number of operating situations (values of the uncertain parameters) taken into account: a trade-off must be made between computation time and the readiness of the system

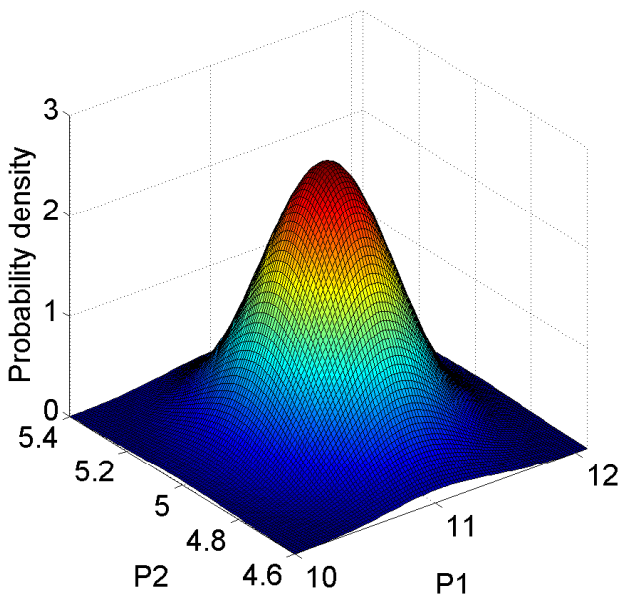

Figure 1: Probability distribution function of a twodimensional Gaussian distribution, corresponding to the uncertainty carried by two loads.

for different operating conditions. Choosing more values from the possible range of the uncertain parameters enables the operator to better prepare the system as more of the uncertainty is considered [13], [14], but it also entails longer computation time. The shorter the considered time horizon the stronger the requirements on short computation times are. Operational decisions require for example faster tools than planning decisions.

- Risk: while solving the SCOPF problem for more operating situations better prepares the system, it does not give any information as for the likelihood of these situations to happen. In other words, the probabilistic information embedded in the probability distributions of the uncertainties (the two loads in Figure 1) is lost. Therefore, the risk associated with different situations is not visible to the system operator.

Chance-constrained optimal power flows address these two shortcomings.

\section{ChancE-CONSTRAINED OPTIMAL POWER FLOWS}

Chance-constrained optimization (CCOPT) problems are optimization problems with probabilistic constraints, also called chance constraints, in which some constraints must hold with a certain probability. These types of problems are particularly adequate for optimization under uncertainty, when some knowledge of the underlying distribution of the uncertainty is known. Compared with deterministic constraints which must always hold, probabilistic constraints allow for some violations with some usually very small pre-defined probability. Two reasons motivate the use of probabilistic constraints when considering uncertainties [5], [15]:

1) When considering rare events for the uncertainty (such as large load or wind power forecast errors in the context of power systems), it might be unavoidable that the constraints are violated. In the theoretical framework, this applies when considering unbounded probability 
distribution function (as is the case with the usual Gaussian distribution for loads), for which there will always be cases, albeit unlikely, for which the constraints are violated.

2) The cost of ensuring satisfaction of the constraints for any event can become exceedingly large, and would not reflect a socio-economic optimum.

Several applications of chance-constrained programming have been made in the context of power systems as described in the introduction. In this work, the formulation and solution method from [9] will be used and compared to the SCOPF formulation in (1). The rest of this section reviews the work from [9].

The chance-constrained optimal power flow (CCOPF) formulation with stability constraints was previously termed stochastic optimal power flow (SOPF) but we rename it here CCOPF to better emphasize the formulation of this optimal power flow as a chance-constrained optimization problem. The CCOPF with stability constraints is defined as

$$
\begin{array}{ll}
\min _{u \in U} & C_{G}(u), \\
\text { s.t. } & p_{\text {fail }}=\sum_{i=0}^{n_{c}} q_{i} P\left[\zeta \notin D_{i}(u)\right] \leq \alpha,
\end{array}
$$

where $u \in U \subset \mathbb{R}^{n_{u}}$ are the control variables, $C_{G}(u): \mathbb{R}^{n_{u}} \rightarrow$ $\mathbb{R}$ is the cost associated with control $u \in U, n_{c}$ is the number of contingencies, $q_{i}$ is the probability that contingency $i$ occurs, $\zeta \in \mathbb{R}^{l}$ are the stochastic system parameters (load and wind power for example), $D_{i}(u) \subset \mathbb{R}^{l}$ is the stable operation domain in $\mathbb{R}^{l}$ and $1-\alpha$ is the desired level of system security. The case $i=0$ corresponds to the pre-contingency system. Contingencies occur with a small probability so that $q_{i} \ll 1$ for $i=1, \ldots, n_{c}$ and $q_{0} \approx 1$.

The optimal solution $u^{*}$ is the optimal re-dispatch of the participating generators which ensures that the probability of system failure is less than a defined $\alpha$. The probability of system failure $p_{\text {fail }}$, defined in the left-hand side of the constraint, is the probability of the operating conditions $\zeta$ to be outside of the stable operation domain. The uncertainty lies in the stochastic system parameters $\zeta$ and in the occurrence of contingencies.

Solving the CCOPF requires the computation of $p_{\text {fail }}$ and its derivatives with respect to $u, \nabla_{u} p_{\text {fail }}$ and $\nabla_{u u} p_{\text {fail }}$. A key point to solve the CCOPF is therefore the characterization of the stable operation domains $D_{i}(u)$ for all pre- and post-contingency systems $i=0, \ldots, n_{c}$. These domains are bounded by the stability boundaries $\Sigma_{i}(u), i=0, \ldots, n_{c}$. A detailed discussion about these stability boundaries is given in [16, Section 5.3]. Each boundary $\Sigma_{i}$ consists in general of different smooth parts $\Sigma_{i j}, j \in J_{i}$. In order to solve (2) analytical expressions of the stability boundaries are needed. However, such expressions do not exist. In [6], it was proposed that each smooth part $\Sigma_{i j}$ of the stability boundaries be approximated by a second-order approximation $\Sigma_{i j}^{a}$. These second-order approximations were presented first in [17] and later developed in [18]-[20]. An extensive description of these approximations is given in [16, Chapter 6]. Using secondorder approximations, the CCOPF problem in (2) can be approximated by

$$
\begin{array}{ll}
\min _{u \in U} & C_{G}(u), \\
\text { s.t. } & \sum_{i=0}^{n_{c}} q_{i} P\left[\min _{j \in J_{i}} d_{i j}(u, \zeta)<0\right] \leq \alpha,
\end{array}
$$

where $d_{i j}, i=0, \ldots, n_{c}, j \in J_{i}$ are distance functions giving the distance from any point $(u, \zeta)$ to the secondorder approximations $\Sigma_{i j}^{a}$, in the direction of the normal to these approximations. The distance functions are defined such that they are positive if the considered point lies beyond the second-order approximations and negative otherwise, which is why the event $\zeta \notin D_{i}(u)$ in the constraint of the original problem (2) is approximated by $\min _{j \in J_{i}} d_{i j}(u, \zeta)<0$ in (3). Note that the distance functions are random variables since $\zeta$ is itself a multivariate random variable.

No analytical formulas exist to express the probability of a minimum such as in (3b). In [6], [9], an approximation scheme for computing the probabilistic constraint and its derivatives is given. The approximation scheme relies on an inclusionexclusion method based on the Hunter-Worsley bound for multivariate probability integrals [21] and on Edgeworth approximations [22]. The problem in (3) then becomes

$$
\begin{array}{ll}
\min _{u \in U} & C_{G}(u), \\
\text { s.t. } & \hat{p}_{\text {fail }}(u)=\sum_{i=0}^{n_{c}} q_{i} \hat{p}^{i}(u) \leq \alpha,
\end{array}
$$

where $\hat{p}^{i}(u)$ is the approximation of $P\left[\min _{j \in J_{i}} d_{i j}(u, \zeta)<0\right]$ based on the approximation scheme from [6], [9].

The problem in (4) is a nonlinear optimization problem. The approximation scheme presented above allows for the computation of both the value $\hat{p}_{\text {fail }}(u)$ of the constraint and its first- and second-order derivatives $\nabla_{u} \hat{p}_{\text {fail }}(u)$ and $\nabla_{u u} \hat{p}_{\text {fail }}(u)$. The Karush-Kuhn-Tucker (KKT) conditions are used to find a local optimum to the problem [23].

The main difference between SCOPF and CCOPF is that the generation re-dispatch given by SCOPF is optimal for a certain chosen outcome $\hat{\zeta}$ of the uncertain parameters (i.e. for chosen operating conditions), while the generation re-dispatch obtained from CCOPF accounts for the whole forecast error distribution of $\zeta$. Furthermore, the probability of system failure is made visible to the system operator when using CCOPF.

\section{CASE STUdY}

\section{A. System setup}

The IEEE 39 bus power system in Figure 2 is considered. All values of the parameters are the same as in [16] and [24]. In this system, generation re-dispatch for a time horizon of 15 minutes will be considered.

The system has 10 generators and 19 loads. All generators are modeled by the one-axis model and equipped with automatic voltage regulators (AVR) with overexcitation limiters 


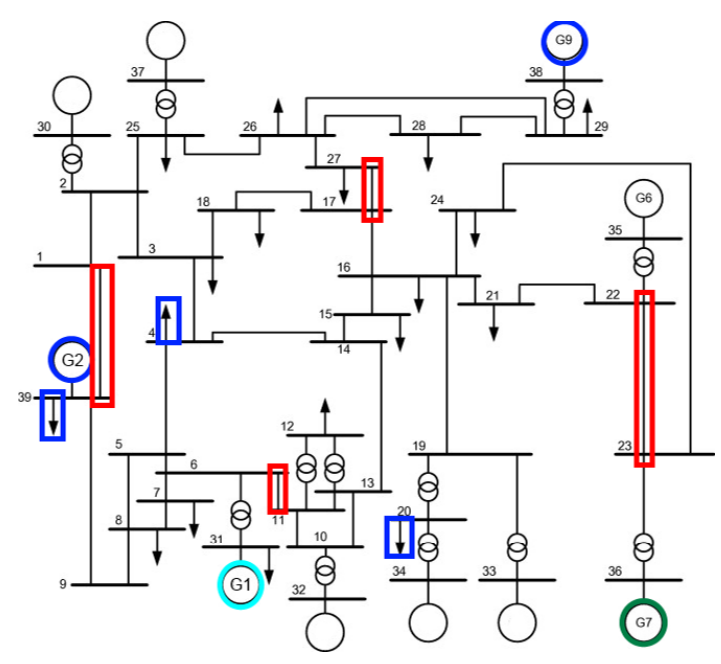

Figure 2: IEEE 39 bus system. The meaning of the circled elements is explained in the text.

Table I: Parameters of the probability distribution

\begin{tabular}{cccccc}
\hline$\mu_{L}$ & $\Sigma_{L}$ & & $a$ & $b$ & $P_{\text {inst }}$ [p.u.] \\
\hline$\left[\begin{array}{c}11.04 \\
5 \\
6.28\end{array}\right]$ & {$\left[\begin{array}{ccc}0.361 & 0 & 0 \\
0 & 0.1635 & 0 \\
0 & 0 & 0.2054\end{array}\right]$} & 25 & 25 & 5.6 \\
\hline
\end{tabular}

(OXL), which are modeled by the following equations:

$$
\begin{aligned}
f^{a, i}(z) & =E_{f, i}+K_{i}\left(V_{r e f, i}-V_{i}\right), \quad i=1, \ldots, 10 \\
f^{b, i}(z) & =-E_{f, i}+E_{f, i}^{\lim }, \quad i=1, \ldots, 10,
\end{aligned}
$$

$E_{f, i}$ are the excitation field voltages, $K_{i}$ the gains of the exciters, $V_{r e f, i}$ the terminal voltage references, $V_{i}$ the terminal voltages and $E_{f, i}^{\lim }$ the limits of the exciters.

Generator 1, circled in cyan in Figure 2 is taken as the slack bus. Generator 7, circled in green in Figure 2, is considered to be a wind farm whose installed capacity is $P_{\text {inst }}=560 \mathrm{MW}$ and whose production is $P_{g 7}$. Generator 2 and 9, circled in blue in Figure 2, participate in generation re-dispatch. The power productions of the other generators are fixed to the values given in [24].

The loads at buses 2, 14 and 30, circled in blue in Figure 2, are assumed to be independent and Gaussian distributed $\left(\mu_{L}, \Sigma_{L}\right)$, see Table I. The uncertainty $\zeta$ also includes the wind power production in generator $7, P_{g 7}$, $\zeta=\left[\begin{array}{llll}P_{g 7} & P_{L, 2} & P_{L, 14} & P_{L, 30}\end{array}\right]^{T}$, where $P_{g 7}=X P_{\text {inst }}$ with $X$ a $\beta(a, b)$-distributed random variable, see Table I. The other loads are fixed at their values given in [24]. The parameters of the probability distributions are gathered in Table I. The parameters of the beta distributions have been taken following the analysis made in [25], where the 15-minute forecast error for a single wind plant is fitted to a similar beta distribution. The base power for the per-unit system is $100 \mathrm{MW}$.

Four contingencies are considered: the removals of lines 13 (between buses 27 and 37), 28 (buses 22 and 23), 35 (buses 16 and 31) and 46 (buses 2 and 11). These lines are circled in red in Figure 2.

\section{B. Objective of the study}

In this system and given the studied contingencies, the SCOPF problem in (1) becomes

$$
\begin{aligned}
\min _{v \in \mathbb{R}^{2}} & C_{G}(v) \\
\text { s.t. } & f_{i}\left(x_{i}, \hat{\zeta}, v\right)=0, \quad i=0, \ldots, 4 \\
& h_{i}\left(x_{i}, \hat{\zeta}, v\right) \leq 0, \quad i=0, \ldots, 4
\end{aligned}
$$

where $\hat{\zeta}=\left[\begin{array}{llll}\hat{P}_{g 7} & \hat{P}_{L, 2} & \hat{P}_{L, 14} & \hat{P}_{L, 30}\end{array}\right]^{T}$ corresponds to the value of the uncertainty for which the SCOPF is solved, and $v=\left[\begin{array}{ll}u_{2} & u_{9}\end{array}\right]^{T}$ corresponds to the re-dispatch orders to generators 2 and 9 , whose power productions are $P_{g, 2}=P_{g, 2}^{0}+u_{2}$ and $P_{g, 9}=P_{g, 9}^{0}+u_{9}$. We assume here $P_{g, 2}^{0}=P_{g, 9}^{0}=0$ so that $P_{g, 2}=u_{2}$ and $P_{g, 9}=u_{9}$. In this example, $f_{i}$ includes the power flow equations, the steady-state one-axis model equations, and for each generator $j, j=1, \ldots, 10$, the product $f^{a, j} \cdot f^{b, j}$ in (5). The equations in $h_{i}$ are all $-f^{a, j}$ and $-f^{b, j}$ for all generators $j=1, \ldots, 10$.

The approximation of the CCOPF problem in this system is

$$
\begin{array}{ll}
\min _{v \in \mathbb{R}^{2}} & C_{G}(v), \\
\text { s.t. } & \hat{p}_{\text {fail }}(v)=q_{0} \hat{p}^{0}(v)+\sum_{j=1}^{4} q_{j} \hat{p}^{j}(v) \leq \alpha,
\end{array}
$$

where $\hat{p}^{0}$ and $\hat{p}^{j}, j=1, \ldots, 4$ are the estimated probability of system failure in the pre- and the four post-contingency systems, respectively. These probabilities depend on the probabilistic forecasts of the uncertainty $\zeta$. Compared to the SCOPF problem which only considers a point forecast $\hat{\zeta}$, the CCOPF problem considers the whole forecast error distribution. Furthermore, the failure rates $q_{i}, i=1, \ldots, 4$ associated with each contingency are considered, and each set to 0.01 . The corresponding value for the pre-contingency case is set to $q_{0}=0.96$.

The cost functions $C_{G}(v)$ in (6) and (7) are the same and are defined by

$$
C_{G}\left(u_{2}, u_{9}\right)=0.6+0.3 u_{2}+0.6 u_{9}+0.01 u_{2}^{2}+0.02 u_{9}^{2} .
$$

The objective of this case study is to compare the optimal re-dispatch solutions obtained by SCOPF in (6) and by CCOPF in (7), given the uncertainty in the loads and wind power production and the selected contingencies. The comparison is done as follows.

1) Different values $\hat{\zeta}_{k}$ of the parameters $\zeta$, corresponding to different possible future operating conditions, are chosen. These operating conditions can be found in Table II.

2) The SCOPF problem is solved for each of these operating conditions, resulting in an optimal re-dispatch $\hat{v}_{k}=\left[\begin{array}{ll}\hat{u}_{2}^{k} & \hat{u}_{9}^{k}\end{array}\right]^{T}$ of generators 2 and 9, with associated $\operatorname{cost} \hat{C}_{k}$.

3) Generators 2 and 9 are set according to $\hat{v}_{k}$ and the corresponding probability of system failure $\alpha_{k}=\hat{p}_{\text {inst }}\left(\hat{v}_{k}\right)$ in (7b) with these generation levels is computed. 
Table III: Comparison of the optimal generation re-dispatch using SCOPF and CCOPF

\begin{tabular}{|c|c|c|c|c|c|c|c|c|c|c|c|}
\hline \multirow[b]{2}{*}{$\begin{array}{c}\text { SCOPF } \\
\text { scenario \# }\end{array}$} & \multirow[b]{2}{*}{$P_{g, 2}$} & \multicolumn{3}{|c|}{ SCOPF } & \multicolumn{7}{|c|}{ CCOPF } \\
\hline & & $P_{g, 9}$ & $P_{g, 2}+P_{g, 9}$ & Cost $\hat{C}$ & $\begin{array}{c}\text { Probability } \\
\text { of system } \\
\text { failure } \\
\hat{p}_{\text {fail }}(\hat{v})\end{array}$ & $P_{g, 2}$ & $P_{g, 9}$ & $P_{g, 2}+P_{g, 9}$ & Cost $C^{*}$ & $\begin{array}{c}\text { Probability } \\
\text { of system } \\
\text { failure } \\
\hat{p}_{\text {fail }}\left(v^{*}\right)\end{array}$ & $\begin{array}{c}\text { Difference } \\
\text { in cost } \\
\hat{C}-C^{*}\end{array}$ \\
\hline 1 & 14.234 & 1.401 & 15.635 & 7.776 & $3.657 \mathrm{e}-01$ & 14.234 & 1.401 & 15.635 & 7.776 & $3.657 \mathrm{e}-01$ & 0 \\
\hline 2 & 14.898 & 1.492 & 16.389 & 8.228 & $6.002 \mathrm{e}-02$ & 14.510 & 1.830 & 16.340 & 8.223 & $6.001 \mathrm{e}-02$ & 0.00492 \\
\hline 3 & 15.253 & 1.869 & 17.121 & 8.693 & $4.620 \mathrm{e}-03$ & 14.610 & 2.350 & 16.960 & 8.638 & $4.590 \mathrm{e}-03$ & 0.0553 \\
\hline 4 & 15.720 & 2.157 & 17.877 & 9.174 & $1.723 \mathrm{e}-03$ & 14.710 & 2.480 & 17.190 & 8.788 & $1.715 \mathrm{e}-03$ & 0.3865 \\
\hline 5 & 14.627 & 1.408 & 16.035 & 8.012 & $1.632 \mathrm{e}-01$ & 14.420 & 1.590 & 16.010 & 8.010 & $1.630 \mathrm{e}-01$ & 0.00197 \\
\hline 6 & 14.863 & 1.560 & 16.423 & 8.253 & $5.234 \mathrm{e}-02$ & 14.520 & 1.860 & 16.380 & 8.249 & $5.221 \mathrm{e}-02$ & 0.00326 \\
\hline 7 & 15.011 & 1.796 & 16.806 & 8.498 & $1.254 \mathrm{e}-02$ & 14.660 & 2.090 & 16.750 & 8.489 & $1.253 \mathrm{e}-02$ & 0.00983 \\
\hline 8 & 15.442 & 2.065 & 17.507 & 8.942 & $2.226 \mathrm{e}-03$ & 14.900 & 2.290 & 17.190 & 8.769 & $2.213 \mathrm{e}-03$ & 0.173 \\
\hline 9 & 13.419 & 1.452 & 14.871 & 7.340 & $8.241 \mathrm{e}-01$ & 13.710 & 1.180 & 14.890 & 7.328 & $8.239 \mathrm{e}-01$ & 0.0113 \\
\hline 10 & 13.895 & 1.380 & 15.274 & 7.565 & $5.975 \mathrm{e}-01$ & 13.895 & 1.380 & 15.274 & 7.565 & $5.975 \mathrm{e}-01$ & 0 \\
\hline
\end{tabular}

4) For each $\alpha_{k}$, the CCOPF in (7) is solved, resulting in an optimal re-dispatch $v_{k}^{*}$ with associated cost $C_{k}^{*}$. The optimal re-dispatch from the CCOPF will therefore ensure the same level of system security $1-\alpha_{k}$ as the optimal re-dispatch from the SCOPF.

5) The optimal re-dispatches $\hat{v}_{k}$ and $v_{k}^{*}$, and their associated costs, $\hat{C}_{k}$ and $C_{k}^{*}$ are compared.

\section{Solution scheme}

In this small example, the following is done to solve the CCOPF problem. A grid of values for $P_{g, 2}$ and $P_{g, 9}$ is set up. For each point $(i, j)$ of the grid corresponding to $\left(P_{g, 2}^{i}, P_{g, 9}^{j}\right)$, the probability of system failure $\hat{p}_{\text {fail }}^{i, j}$ and the cost are computed. When solving CCOPF for $\alpha_{k}$ (step 4 above), the optimal generation re-dispatch is obtained by choosing the point $(i, j)$ with minimal cost which ensures a probability of system failure smaller than $\alpha_{k}$. Therefore, the probability of system failure associated with the CCOPF solution will be equal to that of the SCOPF, up to the grid resolution. Also, because the optimal re-dispatch from SCOPF is also a feasible solution of the CCOPF problem, the costs of the CCOPF solutions will be smaller than that of the SCOPF solutions.

\section{Results}

In Table III, the optimal re-dispatches, costs, and associated probabilities of failure can be found for both SCOPF and

Table II: Operating conditions for which SCOPF is solved

\begin{tabular}{ccccc}
\hline $\begin{array}{c}\text { SCOPF } \\
\text { scenario } \\
\#\end{array}$ & $\begin{array}{c}\text { Wind power } \\
P_{g 7}\end{array}$ & $P_{L, 2}$ & $\begin{array}{c}\text { Loads } \\
P_{L, 14}\end{array}$ & $P_{L, 30}$ \\
\hline 1 & & & & \\
2 & 2.80 & 11.04 & 5.00 & 6.28 \\
3 & 2.80 & 11.40 & 5.16 & 6.49 \\
4 & 2.80 & 11.76 & 5.33 & 6.69 \\
5 & 2.80 & 12.12 & 5.49 & 6.90 \\
6 & 2.41 & 11.04 & 5.00 & 6.28 \\
7 & 2.02 & 11.04 & 5.00 & 6.28 \\
8 & 1.62 & 11.04 & 5.00 & 6.28 \\
9 & 2.41 & 11.76 & 5.33 & 6.69 \\
10 & 2.80 & 10.68 & 4.84 & 6.07 \\
\hline
\end{tabular}

CCOPF. For example, row 4 corresponds to the 4 th SCOPF scenario in Table II. Once the optimal generation re-dispatch from SCOPF was obtained, the CCOPF was solved with $\alpha_{4}=1.723 \cdot 10^{-3}$, which is the probability of system failure associated with the SCOPF re-dispatch. The last column shows the differences between the SCOPF dispatch costs and the CCOPF dispatch costs.

The results show that the more stressed the system is chosen to be when solving SCOPF, the lower the probability of system failure associated with the optimal re-dispatch from SCOPF. It can be seen by comparing the first four scenarios in which the loads used in SCOPF are gradually increased. Since the optimal re-dispatch volumes then increase (because more generation is needed to guarantee system stability when loads are higher), the system will remain stable for a larger range of operating conditions. Therefore, the probability of system failure decreases. The same conclusions can be drawn from scenarios 5 to 7 in which the production in the wind farm in node 7 is gradually decreased from its expected value, thus forcing the controllable generators 2 and 9 to produce more in the optimal solution to keep the system secure. Scenarios 8 to 10 correspond to combined decreases or increases in the loads and wind power production used when solving the SCOPF. We observe that both the costs and the probabilities of system failure of the CCOPF solutions are smaller than that of the SCOPF solutions, as expected.

\section{Discussion}

The gains in dispatch cost vary between the different scenarios as seen in Table III. The gains are high for scenarios 4 and 8 and small for the others. In larger systems with more controllable generators, the gains can be expected to be larger, since the space of feasible solutions for the CCOPF will be augmented. Conversely, with only one controllable generator, the optimal solutions from SCOPF and CCOPF would be the same since there is a one-to-one correspondence between the level of generation in the controllable generator and the probability of system failure [9]. Another advantage of using CCOPF is that the probability of system failure is made visible to the system operator. 
The solution scheme in the small example above was used for illustration purposes. In practice, as explained in Section III, a gradient-based approach, using the first- and secondorder derivatives of the probability of system failure, is used to find the optimal solution, as in [6].

\section{CONCLUSION}

Two methods for short-term generation re-dispatch are compared in this paper. The first method is the classical securityconstrained optimal power flow which optimizes the production of controllable generators for given operating conditions. The second method is the chance-constrained optimal power flow, which optimizes the production of controllable generators for a given probability distribution of the uncertainty and ensures a given level of system reliability.

The two methods are used to find optimal re-dispatch of two generators in the IEEE 39 bus system where the uncertainty from one wind farm and three loads was considered. Through this case study, the benefits of using the chance-constrained optimal power flow are highlighted, namely that it considers the whole information given by the probabilistic forecasts and optimizes the production levels accordingly to ensure a given level of system reliability. Compared to using generation redispatch from SCOPFs, it allows for a better use of the system resources to meet the future operating conditions considered in the forecasts.

\section{REFERENCES}

[1] T. Heidel, J. Kassakian, and R. Schmalensee, "Forward Pass: Policy Challenges and Technical Opportunities on the U.S. Electric Grid", IEEE Power and Energy Magazine, vol. 10, no. 3, pp. 30-37, May 2012.

[2] M. Lauby, M. Ahlstrom, D. Brooks, S. Beuning, J. Caspary, W. Grant, B. Kirby, M. Milligan, M. O’Malley, M. Patel, R. Piwko, P. Pourbeik, D. Shirmohammadi, and J. Smith, "Balancing Act", IEEE Power and Energy Magazine, vol. 9, no. 6, pp. 75-85, Nov. 2011.

[3] J. C. Smith, S. Beuning, H. Durrwachter, E. Ela, D. Hawkins, B. Kirby, W. Lasher, J. Lowell, K. Porter, K. Schuyler, and P. Sotkiewicz, "The Wind at Our Backs", IEEE Power and Energy Magazine, vol. 8, no. 5, pp. 63-71, Sep. 2010.

[4] I. Dobson, S. Greene, R. Rajaraman, C. L. DeMarco, F. L. Alvarado, M. Glavic, J. Zhang, and R. Zimmerman, "Electric Power Transfer Capability: Concepts, Applications, Sensitivity, Uncertainty", Power Systems Engineering Research Center, Technical report 01-34, Nov. 2001.

[5] H. Zhang and P. Li, "Chance Constrained Programming for Optimal Power Flow Under Uncertainty", IEEE Transactions on Power Systems, vol. 26, no. 4, pp. 2417-2424, Nov. 2011.

[6] M. Perninge and C. Hamon, "A Stochastic Optimal Power Flow Problem With Stability Constraints, Part II: The Optimization Problem", IEEE Transactions on Power Systems, vol. 28, no. 2, pp. 1849-1857, 2013.

[7] H. Bludszuweit, J. Dominguez-Navarro, and A. Llombart, "Statistical Analysis of Wind Power Forecast Error", IEEE Transactions on Power Systems, vol. 23, no. 3, pp. 983-991, Aug. 2008.

[8] B. Hodge, D Lew, M Milligan, and H Holttinen, "Wind Power Forecasting Error Distributions: An International Comparison", in 11th Annual International Workshop on Large-Scale Integration of Wind Power into Power Systems as well as on Transmission Networks for Offshore Wind Plants, 2012.
[9] C. Hamon, M. Perninge, and L. Söder, "Applying stochastic optimal power flow to power systems with large amounts of wind power and detailed stability limits", in Bulk Power System Dynamics and Control (iREP) - IX (iREP), 2013 iREP Symposium, 2013, To be published in August 2013.

[10] O. Alsac and B. Stott, "Optimal Load Flow with SteadyState Security", IEEE Transactions on Power Apparatus and Systems, vol. PAS-93, no. 3, pp. 745-751, May 1974.

[11] A. Monticelli, M. V. F. Pereira, and S. Granville, "SecurityConstrained Optimal Power Flow with Post-Contingency Corrective Rescheduling", IEEE Transactions on Power Systems, vol. 2, no. 1, pp. 175-180, 1987.

[12] F. Capitanescu, T. Van Cutsem, and L. Wehenkel, "Coupling Optimization and Dynamic Simulation for PreventiveCorrective Control of Voltage Instability", IEEE Transactions on Power Systems, vol. 24, no. 2, pp. 796-805, May 2009.

[13] F. Capitanescu and L. Wehenkel, "Computation of Worst Operation Scenarios Under Uncertainty for Static Security Management", Power Systems, IEEE Transactions on, vol. 28, no. 2, pp. 1697-1705, 2013.

[14] F. Capitanescu, J. Martinez Ramos, P. Panciatici, D. Kirschen, A. Marano Marcolini, L. Platbrood, and L. Wehenkel, "Stateof-the-art, challenges, and future trends in security constrained optimal power flow", Electric Power Systems Research, vol. 81, no. 8, pp. 1731-1741, Aug. 2011.

[15] A. Geletu, M. Klöppel, H. Zhang, and P. Li, "Advances and applications of chance-constrained approaches to systems optimisation under uncertainty", International Journal of Systems Science, vol. 44, no. 7, pp. 1209-1232, Jul. 2013.

[16] C. Hamon, "On Frequency Control Schemes in Power Systems with Large Amounts of Wind Power", Licentiate Thesis, KTH, 2012.

[17] M. Perninge and L. Söder, "Optimal distribution of primary control participation with respect to voltage stability", Electric Power Systems Research, 2010.

[18] _ _ "On the Validity of Local Approximations of the Power System Loadability Surface", IEEE Transactions on Power Systems, vol. PP, no. 99, pp. 1-1, Mar. 2011.

[19] M. Perninge, "Approximating the loadability surface in the presence of SNB-SLL corner points", Electric Power Systems Research, vol. 96, pp. 64-74, Mar. 2013.

[20] C. Hamon, M. Perninge, and L. Soder, "A Stochastic Optimal Power Flow Problem With Stability Constraints, Part I: Approximating the Stability Boundary", IEEE Transactions on Power Systems, vol. 28, no. 2, pp. 1839-1848, 2013.

[21] T Szántai, "Approximation of Multivariate Probability Integrals.", in Encyclopedia of Optimization, C. A. Floudas and P. M. Pardalos, Eds., Springer US, 2009, pp. 83-90.

[22] P. McCullagh, Tensor Methods in Statistics. Chapman and Hall, 1987.

[23] I Griva, S. Nash, and A Sofer, "Linear and nonlinear optimization", 2009.

[24] R. Miñano, "Optimal power flow with stability constraints", $\mathrm{PhD}$ thesis, Universidad de Castilla-La Mancha, 2010.

[25] B Hodge and M Milligan, "Wind power forecasting error distributions over multiple timescales", Power and Energy Society General Meeting, 2011 IEEE, 2011. 